\title{
Improving Information Ranking by Respecting the Multidimensionality and Uncertainty of User Preferences
}

\author{
Bettina Berendt and Veit Köppen
}

\begin{abstract}
Rankings and ratings are popular methods for structuring large information sets in search engines, e-Commerce, e-Learning, etc. But do they produce the right rankings for their users? In this paper, we give an overview of major evaluation approaches for rankings as well as major challenges facing the use and usability of rankings. We point out the importance of an interdisciplinary perspective for a truly user-centric evaluation of rankings. We then focus on two central problems: the multidimensionality of the criteria that influence both users' and systems' rankings, and the randomness inherent in users' preferences. We propose multi-criteria decision analysis and the integration of randomness into rankings as solution approaches to these problems. We close with an outlook on new challenges arising for ranking when systems address not only individuals, but also groups.
\end{abstract}

\section{Introduction}

Rankings and ratings are popular methods for structuring large information sets such as search engine results or products or documents in e-Commerce, e-Learning, and other environments. Their details are highly dependent on the intentions of suppliers and users. Research on ranking ${ }^{1}$ investigates the underlying concepts and axioms,

Bettina Berendt

K.U.Leuven, Dept. of Computer Science, Leuven, Belgium http://www.cs.kuleuven. be/ berendt

Veit Köppen

Otto-von-Guericke-Universität Magdeburg, Dept. of Computer Science, Magdeburg, Germany http://www.veit-koeppen.de/

${ }^{1}$ Throughout this paper, we will regard ratings as a subclass of rankings. This is done because both share many structural features, see Section 2, and because typical ranking applications such as search engines and typical rating applications such as recommender systems also share many deployment and usage issues. Of course, there are also differences between the two; we will highlight an important source of such differences in Section 2.2. 
cf. (Altman and Tennenholtz 2008; Marchant 2009; Rousseau 2008), as well as suitable methods such as PageRank (Langville and Meyer 2006) and other algorithms for search engines (Chakrabarti 2003). However, a focus on formal and computational aspects is often accompanied by a neglect of the challenges posed by how ranking systems are actually deployed and used.

The underlying idea of many current approaches is to reduce the inherent complexity of the problem of finding the right ranking, and/or to hide this complexity from the user. In this paper, we describe approaches that instead aim to show complexity and make it manageable. We believe that such approaches, which give more control to users, will ultimately fare better at empowering them than progressively simpler interfaces 'hiding' progressively opaque machine intelligence. ${ }^{2}$ In this paper, we will first, in Section 2, briefly review the simple model of evaluation, some important challenges to it, and some solution proposals discussed in (Berendt 2009). We will refer to search engines and recommender systems as two highly popular classes of applications, emphasizing their commonalities, but also mentioning differences. We will add an important further challenge: users' preferences may not be simple list or set structures. Instead, they may be only partially ordered structures, and they may exhibit random elements. In Section 3, we will then describe two classes of approaches for changing the search experience that address several of these challenges at once: multi-criteria ranking and ranking with randomness. We derive these approaches from theoretical considerations. However, a deployment of these ideas could build on related ideas that are today already implemented in some search engines. We close with an outlook on future research.

\section{Challenges for evaluating rankings}

In this section, we present eight challenges that arise in the context of rankings. First, we introduce a simple model for a ranking system and the user of a ranking. For reasons of space, this section and its references are designed as exemplary rather than as a comprehensive survey. Challenges 1-7 are those identified in (Berendt 2009); challenge 8 was added as a result of discussions emanating from that work.

The list, conceived from the perspective of a Web mining researcher, has a large overlap with another current list (for recommender systems) proposed by Ricci (2008). We focus on those problems that challenge a simple model of evaluation as matching between one system ranking and one user ranking. We disregard questions concerning which user ranking should be predicted; specifically, whether this should be the pre- or post-consumption rating, i.e. the acceptance of the recommendation or the judgment after the user has "consumed" the result. The reason is that we regard this as a meta-challenge - we expect that all challenges concerning

\footnotetext{
${ }^{2}$ The belief in the benefits of more user understanding and control appears to be shared by current major Web sites. For example see Amazon's offer to "view and edit your browsing history" (based on which recommendations are made) and Google's offer to adjust ranking positions or delete items from a search-results list.
} 
the evaluation against one user ranking will also appear for either of a set of user rankings. In addition, we take a different perspective on some application-related questions: Whereas Ricci (2008) considers the building of user trust to be a goal for system development, we regard it as a challenge for evaluation, since trust in one system or brand may distort the idealized situation in which there are independent rankings - that of the system and that of the user. Our list also shares elements with the list of challenges (for information-seeking support systems in general) proposed by Kelly, Dumais, and Pedersen (2009). In particular, our challenges 1 and 2 are subsumed by their "inadequate user and task models", our challenge 3 is part of their "task complexity and evolution", and our challenge 7 also calls for "longitudinal designs". Their fourth challenge, the fact that the Web's dynamic nature continually changes the base of objects available for retrieval, is related to our system dynamics, but not addressed in the current paper. A comprehensive review and integration of these and possible further lists of challenges would be a highly valuable literature review, but is impossible within the scope of the current article.

\section{A simple model of systems, users, and evaluation}

The system and the user have their respective ranking functions. A ranking is generally based on a score, which in turn is normally based on several criteria, and therefore the corresponding ranking function is based on a mapping $f$ from $\mathbb{R}^{m} \rightarrow \mathbb{R}$, where $m$ is the dimensionality of criteria. A scoring function $\Phi: A \rightarrow \mathbb{R}$ on the evaluated alternatives $\alpha \in A$ is given by:

$$
\Phi(\alpha)=f\left(v_{1}\left(x_{1}(\alpha)\right), v_{2}\left(x_{2}(\alpha)\right), \ldots, v_{m}\left(x_{m}(\alpha)\right)\right),
$$

where $v_{i}$ evaluates a feature $x_{i}$ of all the alternatives. With scoring function $\Phi$, a ranking or rating can be constructed. A ranking (function) of $n$ items assigns (usually exactly) one rank from $[1 \ldots n]$ to each of the items; a rating (function) with $k$ "grades" assigns one rating from $[1 \ldots k]$ to each item. Due to the commonalities between the formal derivations of ratings and rankings, we will in the following regard the former as a subclass of the latter. An assumption is that a weighting of the criteria is imposed by $\Phi$. The characteristics of a ranking depend on this function.

A simple starting point for evaluation is to assume that (a) there is a systemgenerated ranking $S$, (b) there is a 'true' user-sided ranking $U$ (usually not known to the user), and (c) evaluation consists of a goodness-of-fit test between $S$ and $U$. There exists a multiplicity of measures for such a comparison, including precision and recall as well as various correlation measures, cf. (Herlocker et al. 2004; Vaughan 2004; Berendt 2009). Performance measure optimization is also under research (Robertson and Zaragoza 2007). However, when the focus is on measures (only), the following challenges are often not sufficiently reflected. 


\subsection{Challenge 1: Context-sensitive tasks}

Partial information often leads to inadequate decisions. For example, a patient may not be willing or able to tell a physician everything about an embarrassing disease in this case, the physician cannot treat the patient adequately. Also in the domain of ranking, partial information can lead to suboptimal decisions. In the case of a Web search, the user does not provide all information that is required to obtain the best ranking. Reasons for these partial provisions may include cost, time, or the concern that overly restrictive queries may lead to zero results, and the consequent issuing of wider queries. Information is also partial on the other side of such a Web search. Not all information may be accessible, e.g., restrictions for robots, or analyzable, e.g., text within graphics. From the user's point of view, performing modified searches in further iterations may improve the results (see also Challenge 3 below). On the system side, new techniques, algorithms, and computational effort are required to improve the information status.

Examples of context properties that may lead to different user preferences are the geographic localization of users as well as linguistic and cultural factors or gender. Various measurements can indicate the values of these properties: IP address, language preference settings, or content choices, cf. for example (Mozilla Labs 2008; Liu and Mihalcea 2007; Hu et al. 2007; Kralisch and Köppen 2005; Kralisch and Berendt 2005; Berendt and Kralisch 2009).

\subsection{Challenge 2: Purpose of using the ranking}

A ranking of the user depends on the purpose of using the ranking. A classical example is a recommender system within a shopping portal that recommends items based on the currently viewed item. For example, users may be told that "people who bought this also bought ...", followed by a list of items sorted by strength of these associations. In this context, items that the user already possesses are not useful recommendations. Other misleading items might result from different categories like audio books instead of a DVD, when the user aims to find dance music. In contrast, users of a search engine who look for, say, their department's Web homepage, may expect to reliably find the same URL every time. Also, a user searching for a dance music band may be pleased to unexpectedly discover that there is also an audio book about that genre.

Solution approaches that improve the ranking use new measures to produce nonobvious recommendations. Novelty brings elements into focus that the user is not familiar with. A formalization of novelty can be achieved by using interaction histories of the user or a quite homogeneous group of users, using publication dates to rank recent elements higher, e.g. (Herlocker et al. 2004). Serendipity ranks items higher that the user might not otherwise have discovered. Herlocker, Konstan, Terveen, and Riedl (2004) and Murakami, Mori, and Orihara (2008) give metrics for measuring serendipity, re-ranking items based on the probability of their usefulness 
for the current user as opposed to their usefulness for all users. An instance of this is the following measure proposed by Murakami, Mori, and Orihara (2008):

$$
\text { relative unexpectedness }=\frac{1}{N} \sum_{i=1}^{N} \max \left\{\operatorname{Pr}\left(s_{i}\right)-\operatorname{Prim}\left(s_{i}\right), 0\right\} \cdot i \operatorname{srel}\left(s_{i}\right) \cdot \frac{\operatorname{count}(i)}{i}
$$

where $\operatorname{Prim}\left(s_{i}\right)$ is the result of a primitive prediction method for the $i$ th item, such as a binary function that returns 1 if $i$ belongs to the user's favorite genre and 0 otherwise, $\operatorname{Pr}\left(s_{i}\right)$ is the result of the used prediction method, and $N$ is the number of items ranked. isrel $\left(s_{1}\right)$ is 1 if the item is related to the user's preferences, and 0 otherwise. count $(i)$ is the number of items suited to the user's preferences lying above the $i$-th rank in the recommendation list.

Zhang and Hurley (2009) address the problem of generating recommendation lists that not only contain novel and relevant items, but also exhibit diversity. They propose a method from economics for assessing inequality, based on concentration curves and an associated index, to analyze the bias of recommendation algorithms against the user's novel preferences. Gollapudi and colleagues (Gollapudi and Sharma 2009; Agrawal et al. 2009) investigate how to introduce diversity into search.

\subsection{Challenge 3: Rankings are results of iterations}

A ranking is often a result of iterations, where the query is adapted by the user to improve the returned item set and its ranking. An improvement for iterative obtained rankings is relevance feedback; cf. the survey in (Ruthven and Lalmas 2003). In each iteration, items are ranked according to their relevance to the user's query (by whichever relevance model is used). The basic idea is to reformulate the query by 'adding' features from items the user selected in the previous round, and 'deleting' features from the items the user did not select. Then, the items get re-ranked according to their relevance to the new query. Technically, a simple form is to compute a new query $Q_{t}$ from the original query $Q$ as follows, where both documents and queries are modeled as term vectors:

$$
Q_{t}=\alpha \cdot Q+\beta \cdot \frac{1}{\left|R_{t}\right|} \sum_{\forall x \in R_{t}} x+\gamma \cdot \frac{1}{\left|N_{t}\right|} \sum_{\forall y \in N_{t}} y,
$$

where $R_{t}$ is the set of relevant items and $N_{t}$ the set of non-relevant items. The parameters $\alpha, \beta$ and $\gamma$, called the "Rocchio coefficients", control the relative impact of each component (Leuski and Allan 2004).

A disadvantage of this simple approach is that an evaluation of relevant items is performed in each iteration, which increases ranking effort. Furthermore, nonobserved items are not treated adequately with this method. However, this assumption is in general not realistic, as Section 2.4 will discuss. 
The repetition of the same or similar queries by different users may be considered another form of iteration. Radlinski and Joachims (2005) propose to learn better rankings from the click-through behavior of previous users with the same information need.

\subsection{Challenge 4: Rankings are only partially perceived}

The system ranking is often only observed partially. Research results on ranking attention, see for instance (Nielsen 2006; Eyetools 2008) support the hypothesis that a ranking is only perceived in the first positions. Whereas the first result is perceived by all users, only about $20 \%$ of users look at the tenth element of the ranking (a generally-found pattern; the concrete numbers are from (Eyetools 2008)). Lewandowski and Höchstötter (2007) observe that this tendency of "information snacking" has increased over the past years. This may be explained by the increased trust of users in search engines' rankings, derived from past interactions with the search engine in which the highly ranked elements were indeed useful. It is also a consequence of well-known patterns in human reading, cf. the observation that newspaper stories "above the fold" are read most, help sell the paper, and therefore are most attractive for advertising.

To address this challenge, evaluation measures that weight the top positions more strongly have been proposed. They include the discounted cumulative gain or the half-life utility metric:

$$
U=\frac{\max \left\{r_{i}-d, 0\right\}}{2^{(i-a) /(a-1)}},
$$

where $r_{i}$ is the user's rating of the item, $d$ is the default rating, and $a$ is the halflife: the rank of the item on the list such that there is a $50 \%$ chance that the user will view that item.

\subsection{Challenge 5: Rankings are embedded in information systems}

The system's ranking $S$ and the user's preferences do not exist in a vacuum. Rather, the user and system form an information system in which IT tries to produce a good approximation of users' needs and present it appropriately. Therefore, the designers of IT systems have to find a usable solution that is accepted (and used) by the users. This is a main requirement for the success of Internet search engines. The search engine usability affects in this context effectiveness, efficiency, and satisfaction.

Usability is defined as the effectiveness, efficiency, and satisfaction with which specified users achieve specified goals in particular environments (Dix et al. 1998), which is captured by ISO 9241 (International Organization for Standardization 2007; International Organization for Standardization 2008). Effectiveness is the ac- 
curacy and completeness with which specified users can achieve specified goals in particular environments. Efficiency denotes the resources expended in relation to the accuracy and completeness of goals achieved. Satisfaction is the comfort and acceptability of the working system. A breakdown by "usability objectives" is helpful to operationalize effectiveness, efficiency and satisfaction metrics (Dix et al. 1998). A comprehensive treatment of usability metrics is given by Tullis and Albert (2008).

For adaptive systems, further usability goals have to be taken into account, cf. (Jameson 2003). An important aspect is the transparency of the systems actions, so the actions are on the one hand understandable and on the other hand predictable for the user. Users should also be in control of what happens in the interaction. (This does not mean that there should be no default values and no system adaptivity. Most users will not want to control all or even the majority of system parameters, but it is usually better if they can do so if they so wish.) Privacy issues have to be respected as well. Last but not least, the system should not limit the users' breadth of experience.

\subsection{Challenge 6: Framing influences user preferences}

Challenge 4 emphasized that the layout of information on the screen may have important influences on whether this information is perceived. However, visual perception is not the only source of bias; the way in which information is phrased is another key determinant of how information is understood - and how preferences are formed.

"Framing" is an inevitable process of selective influence over an individual's perception of the meanings attributed to words or phrases. A frame defines the packaging of a certain piece of content such that certain interpretations are encouraged and others are discouraged. A well-known demonstration of this is due to Tversky and Kahneman (1981), who gave experiment participants two versions of the same facts. The task was to decide between two medical treatments for an epidemic. The "positive frame" described option A as "saving 200 people" and option B as "a onethird probability that 600 people will be saved and a two-thirds probability that no people will be saved". The "negative frame" described option C as "400 people dying" and option D as "a one-third probability that nobody will die and a two-thirds probability that 600 people will die". Even though probabilistically, A and C as well as B and D are equal, people consistently preferred A over B in the positive frame and $\mathrm{D}$ over $\mathrm{C}$ in the negative frame.

Framing effects also occur in Web searches and recommendations. Framing the quest for highly personal information in terms of a gain in personalization makes irrelevant questions seem relevant and non-legitimate questions seem legitimate, cf. (Berendt et al. 2005). Framing a ranked recommendation list as arising from data mining creates the impression of personalization (even if an identical list is presented to everyone), and it leads to a higher willingness to disclose personal information (Kobsa and Teltzrow 2005). 
With regard to document search, framing effects pose a specific challenge: If ranking is based only on the "factual content" of documents, it will miss out on an important source of human preferences of documents over others - recall that in the example, the "factual content" of options A and C was identical, as was that of B and $\mathrm{D}$, but $\mathrm{A}$ was preferred over $\mathrm{C}$ and $\mathrm{D}$ over $\mathrm{B}$. If, on the other hand, documentprocessing techniques such as natural-language understanding were able to extract framing, how should the system deal with it when producing the ranking? Should it try to comply with the user's favorite framing or framing-induced preferences? Or should it try to counteract them, for example by focusing on presenting a diversity of framings? We believe that successful solution approaches should, first and foremost, help people become more aware of the presence of framing and its influences.

Taking a wider perspective, one can observe that the habit of using search engines and other rankings also constitutes a certain frame. It can be expected that this will also influence people's perceptions of rankings. We will investigate this question in the following challenge.

\subsection{Challenge 7: System-use dynamics and erroneous beliefs about algorithms}

Information retrieval and Web search have become such an integral part of everyday activities that there is a strong tendency to (blindly) regard search-engine results as "the truth". To find out whether users relied more on a trusted system's ranking or on their own assessments of the result snippets, Pan, Hembrooke, Joachims, Lorigo, Gay, and Granka (2007) exposed people to one of three experimental conditions: Upon entering a search query, users received either the ranking returned by Google, this ranking with the first and second results exchanged, or this ranking in reversed order. In all three conditions, the interface was the familiar Google result list layout. In all three conditions, users displayed the typical behavior of viewing mainly the first two or three results (see Section 2.4), together with similarly strong preferences in click-through behavior. Although users in the "reversed condition" did appear to notice something unusual (as witnessed by longer viewing times of the results page), they attributed the problems to themselves, stating that they "did not have much luck with several of the questions" or that they "could not think of the right search terms".

This may be regarded as evidence of an exaggerated trust in numbers, fed by a reliance on the "brand" of well-known search engines (Jansen et al. 2007) and by erroneous or naïve beliefs in poorly understood algorithms. The latter phenomenon has been well-studied in another area in which rankings are ubiquitous and used and (often mis-)interpreted especially by non-experts: bibliometrics, see for example (Glänzel 2008). 


\subsection{Challenge 8: User rankings are not simple}

Throughout the previous sections, we have assumed that the user's ranking is a simple list, i.e. a total order. However, research suggests that preference orders such as those described in the economic theory of households (Varian 2007) appear much more often. These orders are only partial. Furthermore, user assessments are often better described by random variables than by fixed rankings.

Solving the conflict of partial orders might be done by using similarity measures that respect partial orders, e.g., corrected pairs as stated by Sørensen, Lerche, and Thomsen (2006). However, a restriction to such changes in measures disregards the other challenges discussed in this paper. We will therefore proceed to discuss partial orders and randomness in connection with these challenges, in particular the multiple dimensions influencing ranking and the need to take an integrated view of the information system in which users, systems, and their rankings interact with each other.

\section{Making multidimensionality transparent}

As the preceding discussion has shown, the "right" ranking is determined by multiple dimensions: text relevance to a query, novelty, geographic proximity of search results, etc. This observation is captured in the basic formulation of ranking functions $\Phi$ (see Equation 1), in the challenges posed by context and purpose (see Sections 2.1 and 2.2), and it may become manifest in the queries issued and results selected in different iterations of a search episode (see Section 2.3).

Not only do multiple dimensions exist, they also have different weights. For example, a simple version of $\Phi$ could be a weighted average of dimension measures. These weights may differ between people, situations, etc. In the following, we will also use criteria as another term to denote dimensions, in order to emphasize that they are criteria for choosing or ranking one alternative over others.

In Section 2, we have concentrated on solution approaches for multidimensionality that focus on helping systems derive the right dimension weights automatically. However, it may well be that a more transparent and user-led control of weights will involve users more strongly and lead to greater satisfaction with the software (see usability criterion "control" in Section 2.5) and/or even lead to better-matching ranking results. It remains to be investigated whether usability/ranking quality is a trade-off and if so, how it is assessed by users. It will certainly make the workings of algorithms more transparent and understandable, thus hopefully reducing blind and erroneous beliefs in algorithms (see Section 2.7).

Special challenges arise when users' multiple criteria are to be taken into account: How can bounds on rationality, such as merely partial preference orders, be taken into account (see Section 2.8)? How can interface design create the best affordances for inspecting and setting criteria and weights (see Sections 2.4 and 2.5)? In 
addition, new questions arise, such as: Can a user-defined criteria setting re-frame the search results or their perception by the search-engine user (see Section 2.6)?

\subsection{Multidimensional Ranking}

To deal with a multitude of criteria, a reduction of dimensionality is nearly always required. This is performed by function $\Phi$, see Eq. 1. This loss of dimensionality might lead to an information loss. Therefore, we present an approach that modifies $f$ via an intermediate mapping $\mathbb{R}^{m} \rightarrow \mathbb{R}^{n}$, where $m \geq n$. ( $\Phi$ remains a mapping from $A$ into $\mathbb{R}$.) The resulting $n$ criteria are used to build a set of rankings, where only one criterion is changed at a time. Note that these $n$ criteria may be aggregated indicators, resulting from transformations of the original $m$ criteria.

The presentation of the top alternatives within a visualization as in Fig. 1 enhances the understanding from the user's viewpoint. The criteria of this example are derived from the observations described in Sections 2.0, 2.1 and 2.5, and their values are shown together with three fictitious alternatives $\alpha_{[1|2| 3]}$ to be ranked. Each criterion is assigned to one spoke of the chart, and for each alternative to be ranked, a line connecting its values on the criteria is drawn. Thus, the chart is a concise way of showing the rankings of all alternatives along all criteria simultaneously. This control of dimensionality with a Kiviat chart is based on the Balanced Scorecard approach proposed and extended by Kaplan and Norton (1996, 2004). The $n$ criteria may be clustered into groups, where different groups are possible. One possible grouping of the criteria of Fig. 1 will be shown below in Fig.3. Navigation through these rankings is necessary due to complexity, and it is possible with visualization techniques. The Kiviat chart is an appropriate choice for such a representation. ${ }^{3}$

The explicit display of different criteria for navigation between alternatives is used in several real-life search tools. It is popular in e-Commerce and other sites that allow users to order the found items by price, popularity, or other criteria; an interaction technique known as faceted search, cf. (Hearst 2006). Berendt and Trümper (2009) define several dimensions of document similarity (textual similarity, namedentity similarity, date similarity, and source class) and provide a visualization of document sets in this space. Documents are arranged in a display plane such that they are visually ordered, along the different criteria, by their similarity to a focus document.

An obvious limitation of a Kiviat chart or other representations that show individual criteria rankings is that often, no alternative will dominate all the others on every criterion. Thus, users may prefer to see a ranking that presents one (preferably total) order on the alternatives, summarizing their rankings along the different criteria. This is the essence of the most commonly used $\Phi$ functions, of which a very simple one will be described next.

${ }^{3}$ Parallel coordinates (d'Ocagne 1885; Inselberg 1985) are information-equivalent to these charts, but they lack the "holistic Gestalt" induced by the circular spoke arrangement of the axes. 


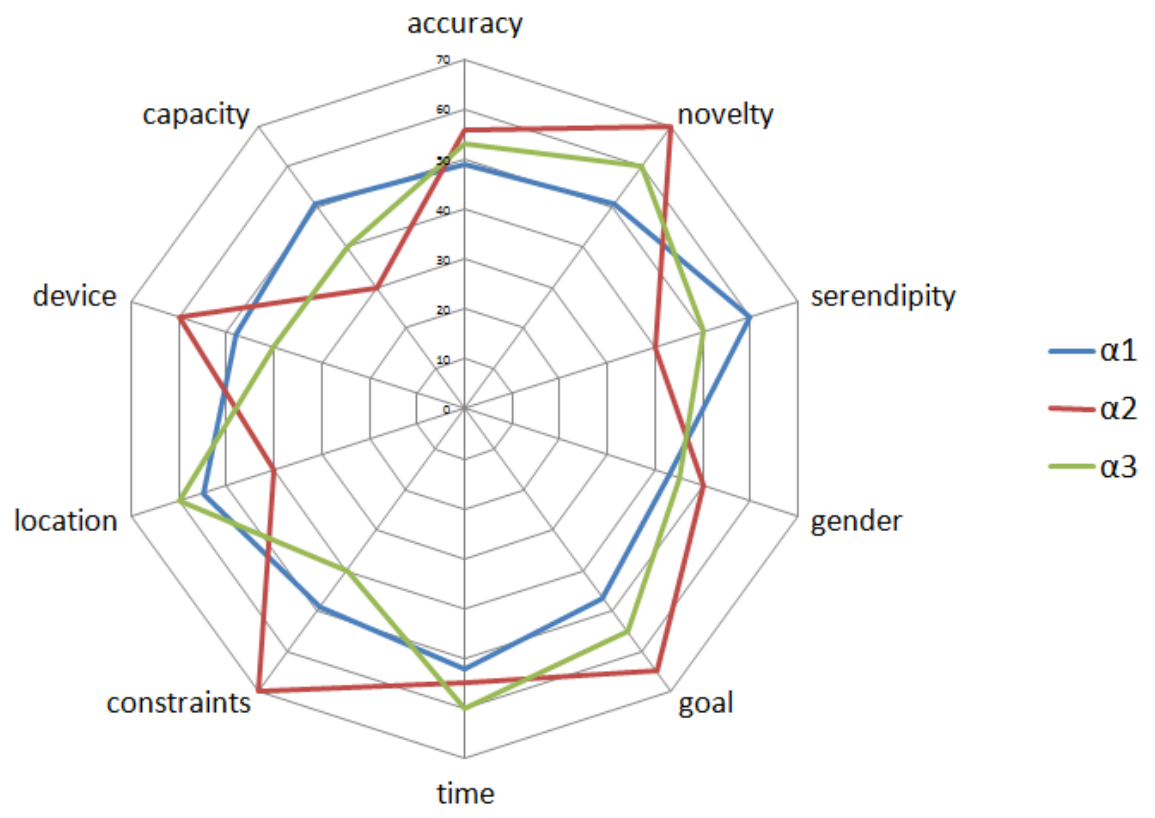

Fig. 1 Kiviat Chart of Selected Ranking Criteria

In Table 1, we extend the example by using a simple $\Phi$ function. The table contains fictitious values $v(x(\alpha))$ that evaluate the three alternatives $\alpha$ from Fig. 1 . We also present two different sets of weights for these criteria and, for each set of weights, a summarizing one-dimensional score and the ranking derived from it. The score is the inner product of the criteria values vector and the weights vector.

Depending on the weights, the obtained ranking differs. For the first set of weights, the ranking is: alternative 2 is followed by 3 and 1 . For the second set of weights, where only small changes in the weights are used, the ranking changes completely. This change is not obvious or traceable for the user if only the ranking is provided - this constitutes the information loss. This may be problematic when the weights are uncertain, and the presentation of a result (based on whichever set of weights) creates a sense of false certainty.

While this is a simple and clear procedure for aggregating different criteria into a ranking, it raises the question of how to acquire those weights. In the following two sections, we will give a brief overview of proposals for addressing these questions. 
Table 1 Evaluation of Three Alternatives $\alpha 1, \alpha 2, \alpha 3$

\begin{tabular}{lccc|cc}
\hline Criterion & $\alpha 1$ & $\alpha 2$ & $\alpha 3$ & Weights 1 & Weights 2 \\
\hline accuracy & 49 & 56 & 53 & 0.10 & 0.05 \\
novelty & 51 & 70 & 60 & 0.10 & 0.05 \\
serendipity & 60 & 40 & 50 & 0.10 & 0.10 \\
gender & 43 & 50 & 45 & 0.10 & 0.10 \\
goal & 47 & 65 & 55 & 0.10 & 0.10 \\
time & 52 & 55 & 60 & 0.10 & 0.10 \\
constraints & 49 & 70 & 40 & 0.10 & 0.10 \\
location & 55 & 40 & 60 & 0.20 & 0.15 \\
device & 48 & 60 & 40 & 0.05 & 0.05 \\
capacity & 51 & 30 & 40 & 0.05 & 0.20 \\
\hline Score 1 & 51.05 & 53.10 & 52.30 & & \\
Ranking 1 & 3 & 1 & 2 & & \\
\hline Score 2 & 50.95 & 49.30 & 49.65 & & \\
Ranking 2 & 1 & 3 & 2 & & \\
\hline
\end{tabular}

\subsection{Weighting the criteria: Multi-Criteria Decision Analysis}

An improvement of the system's ranking can be obtained if the function $\Phi$ of the system is close the the user's one. However, a complete specification is usually not possible due to the complexity and the change over context and time. Therefore, $\Phi$ should be adjusted to make it robust with respect to small temporal changes, as well as lower-dimensional and thus manageable. In a given set of characteristics stated by the system, two possible scenarios are: on the one hand the user might specify an order for each feature, on the other hand the weights of each feature are revealed. Whereas the first possibility is too complex due to the fact that a generation of rankings for each feature is required, the second possibility is much more prudent. In the following approaches are described to obtain these weights.

In this section we use Multi-Criteria Decision Analysis (MCDA) to obtain a ranking where all criteria are respected. MCDA is used to obtain a ranking of alternatives in a decision process. However, it can also be applied for obtaining a ranking in the context of Internet search engines where the decision has to be taken, which of all available elements fits best to a certain information need or query.

In the domain of MCDA, many methods can be differentiated. In Fig. 2 the main approaches are presented according to the classification by Schneeweiß (1991). Further information is given in (Figueira et al. 2005; Bamberg and Coenenberg 2002; Schneeweiß 1991).

A first differentiation can be made on the top level. Methods "with preference functional" are those for which at least a weak preference order of alternatives is available or required. The other approaches do not need such an order. In practice, methods without a preference functional are used when a transitive order of alternatives cannot be found.

A further classification of the preference order functional is also depicted in Fig. 2. When substitution rules between alternatives are given, a "preference function" is available. All alternatives have to be assigned a measurement, and these 
measurements must be interval-scaled. A "preference index" is used if a weak order on alternatives is given. All alternatives might be ordered directly or indirectly via an order on attributes. The distinct evaluation of utility function and weights has to be combined afterwards again to obtain a holistic result. When only a "partial preference functional", i.e. only a partial order relation, is given, iterations of the ranking process are useful. In each iteration, another order relationship is elicited, and this information is used to improve the ranking. However, if a ranking does not differentiate the alternatives sufficiently, a further iteration is required. In all these approaches, a characteristic of an attribute can be compensated by one or more others. A "non-compensating preference functional" orders all alternatives according to the importance of criteria. The ordering is done by considering the most important criterion first and then recursively employing this procedure on all the other criteria. An example is lexicographic ordering.

Methods "without a preference functional", also called outranking, assume that an ordering is not possible or misleading. These approaches are used to find the top solution or a classification of alternatives.

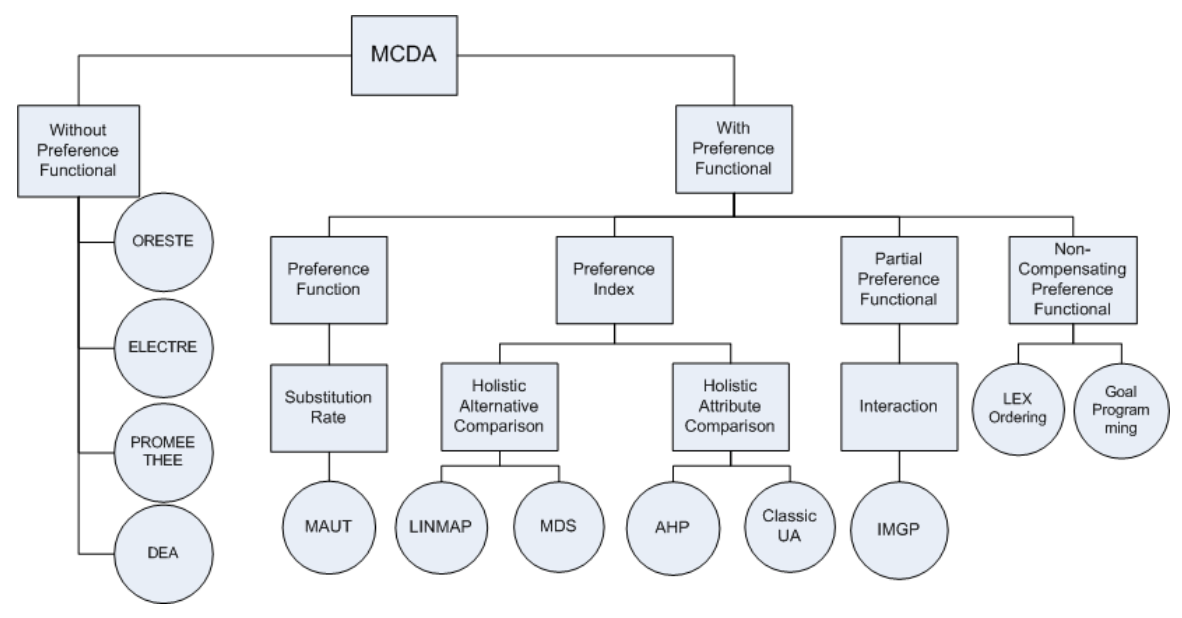

Fig. 2 MCDA Classification, adapted from (Schneeweiß 1991)

In this paper, we restrict ourselves to the Analytic Hierarchy Process as one method with a preference functional, more specifically a preference index. AHP is a method based on "holistic attribute comparison". This means that attributes as a whole are compared to one another irrespective of these attributes' individual values (and possible fine-grained preferences on them). 


\subsection{The Analytic Hierarchy Process method}

The Analytic Hierarchy Process (AHP) (Saaty 1980) is a special case of utility value analysis (Keeney and Raiffa 1976). The weights are holistic and obtained independently of the evaluation function by using pairwise comparisons between attributes. This makes it possible that even if the preference order is inconsistent (non-transitive), the evaluation function is in a consistent state. Therefore, this approach is promising for building rankings with partial orders. However, an important disadvantage of AHP is that when new alternatives are integrated, large and seemingly unmotivated re-rankings can occur. In addition, if the criteria space is high-dimensional, it is costly to obtain all pairwise comparisons.

In the first phase of the AHP, all required criteria are selected and classified into a hierarchy. This classification is used to reduce the pairwise comparisons. A comparison is only necessary for attributes on the same level and with the same parent. In Fig. 3, we present a possible classification for a selection of the criteria introduced above in Fig. 1.

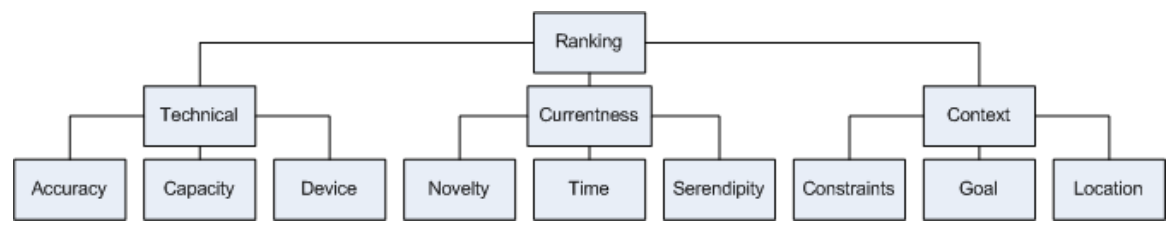

Fig. 3 A Hierarchy for Ranking Criteria

Pairwise comparisons are carried out at each level for nodes with the same parent. In practice, the evaluation is often done using comparison relations like those in Table 2. However, the AHP is not restricted to these relationships.

Table 2 AHP Comparison Values

\begin{tabular}{lr}
\hline Relative Importance & Value \\
\hline equal & 1 \\
somewhat more important & 3 \\
definitely more important & 5 \\
much more important & 7 \\
extremely more important & 9 \\
\hline
\end{tabular}

A pairwise comparison is performed with a quadratic matrix. All diagonal elements are set to 1 , and all known relationships $(i, j)$ are filled in. Then, the missing cells $(j, i)$ are each set to $\frac{1}{(i, j)}$. The result is a set of relative importance tables. A possible relative importance table for the first level of our example from Fig. 3 is shown in Table 3. For example, "Technical" is judged to be "somewhat more important" than "Currentness", thus cells $(1,2)=3$ and $(2,1)=\frac{1}{3}$. 
Table 3 Relative Importance Table for Technical, Currentness, and Context

\begin{tabular}{|c|c|c|c|}
\hline & \multicolumn{3}{|c|}{ Technical Currentness Context } \\
\hline Technical & 1 & 3 & $1 / 5$ \\
\hline Currentness & $1 / 3$ & 1 & $1 / 7$ \\
\hline Context & 5 & 7 & 1 \\
\hline
\end{tabular}

In a simplified version of the computation of the maximum eigenvalue and eigenvectors, the priority vector is obtained (for details, see Saaty 1980). Priority vectors are computed for all criteria and for all alternatives considered for each criterion. Afterwards, the intermediate priority vectors for each criterion are merged with the help of the importance vectors of all criteria, to obtain, for each alternative, a score, which is used to build the ranking.

In an 'ideal world', every user would specify her or his individual preferences on attributes. However, in more realistic settings, a system may at best have such preferences from some users and will have to serve unknown/anonymous users on this basis. Thus, an aggregation over weights becomes necessary. Since the users who expressed preferences as well as those who did not will usually be heterogeneous groups, this can lead to non-appropriate rankings. An example is the aggregation of extreme values towards a mean value, which will please no-one. To solve this conflict, a clustering algorithm can be implemented that resolves heterogeneity.

\section{Making randomness transparent}

As we have argued in Section 2.8, user rankings most probably have an element of randomness. As several studies have shown, users however tend to have a strong belief that system rankings (and therefore the "truth"?!) are deterministic, cf. Section 2.7. As a possible remedy, Pan, Hembrooke, Joachims, Lorigo, Gay, and Granka (2007) suggested in their outlook that ".. [a] certain degree of randomness in the ranking of returned results ... leads to improved search". In this section, we will investigate the possibilities of introducing randomness into rankings in a more principled way.

The first important observation is that this would have to be communicated - randomness would need to be transparent. Otherwise, search engines will risk to appear "buggy", and some user tasks that employ search engines may become unnecessarily difficult. As an example, consider the exchange of the guideline "Google for $\mathrm{XYZ}$ and take the second result". Rankings that present themselves in such a way might also help people remember (more strongly) that context and framing (see Sections 2.1 and 2.4) are ubiquitous and influence rankings.

The randomness of a system's ranking represents the uncertainty of user preferences, which are a result of hidden processes as well as of inconsistencies of the partial ordering. The randomness should therefore be adequately designed, i.e. the parameterization of the underlying distribution function has to be carefully consid- 
ered. The introduction of a random process should be done as an element of the function $\Phi$ as:

$$
\Phi^{*}(\alpha)=\Phi(\alpha)+u
$$

where $u \sim \operatorname{Dist}(\cdot)$ is a random variable distributed according to function Dist. The implementation of randomness in $\Phi^{*}$ should be small so the ranking of the system $S$ is not completely random, but at local points the ranking might differ for different ranking building processes.

Randomness poses further challenges when users want to share rankings with other people, because the ranking is treated as only one possible ordering. A disadvantage of using randomness is that the user does not re-obtain the ranking when issuing the (identical) query again. However, this is only a disadvantage at a first glance: when pseudo-random numbers are used, their generator uses a seed, which determines the "randomness". If this seed is re-used for another ranking building process, the same ranking will be reproduced. This will enable users to re-use their own searches with identical search results, and it will allow them to share these results by sending the user-specific parameters of $\Phi$ including the seed. Obviously, the information thus shared might contain highly privacy-sensitive data: personal preferences together with a seed which, if it is persistent, might be used to uniquely identify the user. Storing and exchanging such information would thus pose new challenges for security and privacy mechanisms.

At present (September 2009), the ranking within result lists shown by Google varies by browser, browser and search-engine preferences (http://www . google. $\mathrm{com} /$ preferences), and possibly other factors. A replication of a result list is possible by saving the URL query string, but users have no further control over this process. This variation may be a fine-tuned response to preferences previously shown, by this or other users, in search episodes with the different browsers - and thus deterministic. However, for many users and usage contexts, the variation is more likely to appear random - regardless of whether or not it is based on deterministic adaptations. One example are users who have to use a browser at work which they do not use at home, but perform identical types of searches in both places: they will probably not expect a search for the evening's cinema program, run at $4 \mathrm{pm}$, while still in the office, to return a different result at $6 \mathrm{pm}$, when repeated from home. Another example are users who run several browsers to obtain the best rendering of different sites, and then start unrelated search episodes in a window that happens to be open: they will probably not understand why there should be a difference between the results in one window (running browser 1) and another (running browser 2). The fact that such variation is effected - whether it be deterministic or random - is at present not easily visible to users. An appropriate estimation of users' acceptance of it, as well as of the randomness of users' preferences, are challenging tasks for future research. 


\section{Conclusion and Outlook}

To evaluate the quality of a ranking such as done in search engines or recommender systems, one must answer the question whether the ranking is the "right ranking"for the given person, in the given circumstances. In this paper, we proposed a very simple general conceptualization of the ranking-evaluation task: the comparison between the ranking generated by a computational system such as a search engine, and the "true ranking inside the user's head". The article then proceeded to describe eight challenges to this simple model. We concluded that they all call for approaches to dealing with multidimensional and often only partial preference orders - both on the part of the users and on the part of the system, and that randomness is probably a characterizing feature of user rankings, and could be a beneficial feature of system rankings. We then proposed that a closer look at existing work in multi-criteria decision analysis and the introduction of randomness into system rankings could address the challenges, thus leading to better ranking systems.

As an outlook, we would like to emphasize an additional challenge: several stakeholders. When the interests and preferences of several stakeholders need to be taken into account, the challenges described above also occur. However, recommendation to groups differs from recommendation to individuals (Jameson and Smyth 2007). One important difference is that group members may want to examine one another's preferences. Therefore, the information about preferences has to be acquired. Another task is the determination of the suitability of items for the group and the generation of recommendations from this. Furthermore, the suitability to different members of the group may be different, and recommendations have to be presented in a way that accommodates such differences. Additionally, for a final decision, negotiation may be necessary or required - so the system must help users arrive at a consensus about which recommendations (if any) are acceptable.

Approaches for solving these subtasks are many-faceted and depend on the intention of the system (or the developer of the system). We will only give some examples of preference aggregation for the generation of multi-user rankings. The group ranking may be derived as the minimum, maximum, or average of the rankings of individual group members. Alternatively, a group preference model may be created, for example as a content profile for the group defined as a linear combination of vector-space representations of Web pages that the members like. Finally, sometimes it may be most adequate to issue a recommendation for the group that is not based on the aggregation of preferences of the group's members. An example is a heuristic like "Walt Disney films are good for families" - regardless of whether any person in the family is a particular fan of such movies. All of these aggregation methods reflect certain strategies and have associated advantages and disadvantages. For example, using the minimum of members' rankings leads to recommending the item that will cause the 'least suffering', while averaging of extreme values may produce a solution that everybody resents as 'a lukewarm compromise'. Many issues in recommendations for groups still need to be resolved. The increasingly Social Web (in which applications interact not only with individuals, but with various collectives) offers many opportunities for such research and application building. 



\section{References}

[Agrawal et al. 2009] Agrawal, R., S. Gollapudi, A. Halverson, and S. Ieong (2009). Diversifying search results. In WSDM '09: Proceedings of the Second ACM International Conference on Web Search and Data Mining, New York, NY, USA, pp. 5-14. ACM.

[Altman and Tennenholtz 2008] Altman, A. and M. Tennenholtz (2008). Axiomatic foundations for ranking systems. Journal of Artificial Intelligence Research 31, 473-495. http://www. jair.org/media/2306/ live-2306-3748-jair.pdf, retrieved 2009-06-15.

[Bamberg and Coenenberg 2002] Bamberg, G. and A. G. Coenenberg (2002). Betriebswirtschaftliche Entscheidungslehre (11 ed.). Munich: Vahlen.

[Berendt 2009] Berendt, B. (2009). Ranking - use and usability. Bulletin of the Belgian Mathematical Society - Simon Stevin 16, 1-24.

[Berendt et al. 2005] Berendt, B., O. Günther, and S. Spiekermann (2005). Privacy in e-commerce: Stated preferences vs. actual behavior. Communications of the ACM 48(4), 101-106.

[Berendt and Kralisch 2009] Berendt, B. and A. Kralisch (2009). A user-centric approach to identifying best deployment strategies for language tools: The impact of content and access language on web user behaviour and attitudes. Journal of Information Retrieval 12(3), 380-399.

[Berendt and Trümper 2009] Berendt, B. and D. Trümper (2009). Semantics-based analysis and navigation of heterogeneous text corpora: The porpoise news and blogs engine. In I.-H. Ting and H.-J. Wu (Eds.), Web Mining Applications in E-commerce and E-services, pp. 45-64. Berlin etc.: Springer.

[Chakrabarti 2003] Chakrabarti, S. (2003). Mining the Web. San Francisco, CA: Morgan Kaufmann.

[Dix et al. 1998] Dix, A., J. Finlay, G. Abowd, and R. Beale (1998). Human Computer Interaction. Prentice Hall Europe.

[d'Ocagne 1885] d'Ocagne, M. (1885). Coordonnées Parallèles et Axiales: Méthode de transformation géométrique et procédé nouveau de calcul graphique déduits de la considération des coordonnées parallèlles. Paris: Gauthier-Villars. 
[Eyetools 2008] Eyetools (2008). Eyetools research and reports: Eyetools, enquiro, and did-it uncover search's golden triangle. http://www. eyetools. com/inpage/research_google_eyetracking_heatmap.htm, retrieved 2009-06-15.

[Figueira et al. 2005] Figueira, J., S. Greco, and M. Erhgott (Eds.) (2005). Multiple Criteria Decision Analysis: State of the art surveys. Boston: Springer Science and Business Media.

[Glänzel 2008] Glänzel, W. (2008). Seven myths in bibliometrics. About facts and fiction in quantitative science studies. Collnet Journal of Scientometrics and Information Management 2(1), 9-17. Conference version at http://www . collnet.de/Berlin-2008/GlanzelWIS2008smb.pdf, retrieved 2009-06-15.

[Gollapudi and Sharma 2009] Gollapudi, S. and A. Sharma (2009). An axiomatic approach for result diversification. In J. Quemada, G. León, Y. S. Maarek, and W. Nejdl (Eds.), WWW, pp. 381-390. ACM.

[Hearst 2006] Hearst, M. A. (2006). Design recommendations for hierarchical faceted search interfaces. In SIGIR'2006 Faceted Search Workshop. http://flamenco.berkeley.edu/papers/ faceted-workshop06.pdf, retrieved 2009-06-15.

[Herlocker et al. 2004] Herlocker, J. L., J. A. Konstan, L. G. Terveen, and J. T. Riedl (2004). Evaluating collaborative filtering recommender systems. ACM Trans. Inf. Syst. 22(1), 5-53.

[Hu et al. 2007] Hu, J., H.-J. Zeng, H. Li, C. Niu, and Z. Chen (2007). Demographic prediction based on user's browsing behavior. In $W W W$ '07: Proceedings of the 16th international conference on World Wide Web, pp. 151-160. ACM.

[Inselberg 1985] Inselberg, A. (1985). The plane with parallel coordinates. Visual Computer 1(4), 69-91.

[International Organization for Standardization 2007] International Organization for Standardization (2007). ISO 9241-400:2007. ergonomics of humansystem interaction - part 400: Principles and requirements for physical input devices. http://www.iso.org/iso/iso_catalogue/ catalogue_tc/catalogue_detail.htm?csnumber=38896, retrieved 2009-06-15.

[International Organization for Standardization 2008] International Organization for Standardization (2008). ISO 9241-151:2008. ergonomics of humansystem interaction - part 151: Guidance on world wide web user interfaces. http://www.iso.org/iso/iso_catalogue/catalogue_tc/ catalogue_detail.htm?csnumber=37031, retrieved 2009-06-15.

[Jameson 2003] Jameson, A. (2003). Adaptive interfaces and agents. In J. A. Jacko and A. Sears (Eds.), Human-Computer Interaction Handbook, pp. 305-330. Mahwah, NJ: Erlbaum. http://dfki.de/ jameson/abs/ Jameson 03Handbook . html, retrieved 2009-06-15.

[Jameson and Smyth 2007] Jameson, A. and B. Smyth (2007). Recommendation to groups. In P. Brusilovsky, A. Kobsa, and W. Nejdl (Eds.), The Adaptive Web, Volume 4321 of Lecture Notes in Computer Science, pp. 596-627. Springer. 
[Jansen et al. 2007] Jansen, B. J., M. Zhang, and Y. Zhang (2007). Brand awareness and the evaluation of search results. In $W W W$ '07: Proceedings of the 16th international conference on World Wide Web, New York, NY, USA, pp. 1139-1140. ACM.

[Kaplan and Norton 1996] Kaplan, R. S. and D. P. Norton (1996). The Balanced Scorecard. Translating Strategy Into Action. Harvard Business School Press.

[Kaplan and Norton 2004] Kaplan, R. S. and D. P. Norton (2004). Strategy Maps: Converting Intangible Assets Into Tangible Outcomes. Harvard Business School Press.

[Keeney and Raiffa 1976] Keeney, R. and H. Raiffa (1976). Decisions with Multiple Objectives; Preferences and Value Tradeoffs. John Wiley \& Sons.

[Kelly et al. 2009] Kelly, D., S. Dumais, and J. O. Pedersen (2009). Evaluation challenges and directions for information-seeking support systems. Computer 42(3), 60-66.

[Kobsa and Teltzrow 2005] Kobsa, A. and M. Teltzrow (2005). Impacts of contextualized communication of privacy practices and personalization benefits on purchase behavior and perceived quality of recommendation. In Beyond Personalization 2005: A Workshop on the Next Stage of Recommender Systems Research (IUI 2005), San Diego, CA, pp. 48-53.

[Kralisch and Berendt 2005] Kralisch, A. and B. Berendt (2005). Languagesensitive search behaviour and the role of domain knowledge. The New Review of Hypermedia and Multimedia 11(2), 221-246.

[Kralisch and Köppen 2005] Kralisch, A. and V. Köppen (2005). The impact of language on website use and user satisfaction: Project description. In Proceedings of the 13th European Conference on Information Systems, Information Systems in a Rapidly Changing Economy, ECIS 2005.

[Langville and Meyer 2006] Langville, A. N. and C. D. Meyer (2006). Google's PageRank and Beyond: The Science of Search Engine Rankings. Princeton University Press.

[Leuski and Allan 2004] Leuski, A. and J. Allan (2004). Interactive information retrieval using clustering and spatial proximity. User Model. User-Adapt. Interact. 14(2-3), 259-288.

[Lewandowski and Höchstötter 2007] Lewandowski, D. and N. Höchstötter (2007). Web searching: A quality measurement perspective. In A. Spink and M. Zimmer (Eds.), Web Searching: Interdisciplinary Perspectives. Dordrecht, The Netherlands: Springer.

[Liu and Mihalcea 2007] Liu, H. and R. Mihalcea (2007). Of men, women, and computers: Data-driven gender modeling for improved user interfaces. In Proceedings of the International Conference on Weblogs Social Media (ICWSM), pp. 121-128.

[Marchant 2009] Marchant, T. (2009). An axiomatic characterization of the ranking based on the $h$-index and some other bibliometric rankings of authors. Scientometrics 80(2), 325-342. 
[Mozilla Labs 2008] Mozilla Labs (2008). Introducing geode. http:// labs. mozilla.com/2008/10/introducing-geode/, retrieved 200906-15.

[Murakami et al. 2008] Murakami, T., K. Mori, and R. Orihara (2008). Metrics for evaluating the serendipity of recommendation lists. In New Frontiers in Artificial Intelligence, JSAI 2007 Conference and Workshops, Miyazaki, Japan, June 18-22, 2007, Revised Selected Papers, Volume 4914 of Lecture Notes in Computer Science, pp. 40-46. Springer.

[Nielsen 2006] Nielsen, J. (2006). Eyetracking research. http://www . useit. com/ eyet racking, retrieved 2009-06-15.

[Pan et al. 2007] Pan, B., H. Hembrooke, T. Joachims, L. Lorigo, G. Gay, and L. Granka (2007). In google we trust: Users' decisions on rank, position, and relevance. Journal of Computer-Mediated Communication 12(3), 801-823.

[Radlinski and Joachims 2005] Radlinski, F. and T. Joachims (2005). Query chains: learning to rank from implicit feedback. In R. Grossman, R. J. Bayardo, and K. P. Bennett (Eds.), Proceedings of the Eleventh ACM SIGKDD International Conference on Knowledge Discovery and Data Mining, pp. 239-248. ACM.

[Ricci 2008] Ricci, F. (2008). Recommender systems. Invited Talk at Intelligent Information Access, Cagliaria, Italy, 10 Dec 2008, urlhttp://iasc2.diee.unica.it/iia2008/index.php/program/.

[Robertson and Zaragoza 2007] Robertson, S. and H. Zaragoza (2007). On rankbased effectiveness measures and optimization. Inf. Retr. 10(3), 321-339.

[Rousseau 2008] Rousseau, R. (2008). Woeginger's axiomatisation of the $h$-index and its relation to the $g$-index, the $h^{(2)}$-index and the $R^{2}$-index. Journal of Informetrics 2(4), 335-340.

[Ruthven and Lalmas 2003] Ruthven, I. and M. Lalmas (2003). A survey on the use of relevance feedback for information access systems. Knowl. Eng. Rev. 18(2), 95-145.

[Saaty 1980] Saaty, T. (1980). The Analytic Hierarchy Process for Decisions in a Complex World. McGraw-Hill.

[Schneeweiß 1991] Schneeweiß, C. (1991). Planung 1, Systemanalytische und entscheidungstheoretische Grundlagen. Berlin: Springer.

[Sørensen et al. 2006] Sørensen, P., D. Lerche, and M. Thomsen (2006). Developing decision support based on field data and partial order theory, pp. 259283. Berlin etc.: Springer.

[Tullis and Albert 2008] Tullis, T. and W. Albert (2008). Measuring the User Experience: Collecting, Analyzing, and Presenting Usability Metrics. Morgan Kaufmann.

[Tversky and Kahneman 1981] Tversky, A. and D. Kahneman (1981). The framing of decisions and the psychology of choice. Science 211, 453-458.

[Varian 2007] Varian, H. R. (2007). Intermediate Microeconomics: A Modern Approach (7 ed.). W W Norton \& Co. 
[Vaughan 2004] Vaughan, L. (2004). New measurements for search engine evaluation proposed and tested. Information Processing \& Management 40(4), 677-691.

[Zhang and Hurley 2009] Zhang, M. and N. Hurley (2009). Statistical modeling of diversity in top-n recommender systems. In Proceedings of the ACM Web Intelligence Conference WI'09, pp. 490-497. IEEE Computer Society. 